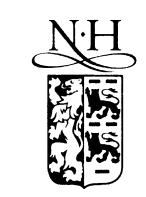

ELSEVIER

\title{
On-line perturbed angular correlation studies with the short lived ${ }^{127}$ Cs probe
}

\author{
J.G. Correia ${ }^{\text {a,b }}$, J.G. Marques ${ }^{\text {a,c }}$, A.A. Melo ${ }^{\text {a }}$, J.C. Soares ${ }^{\text {a,c,* }}$, H. Haas ${ }^{\text {d, }}$, \\ ISOLDE Collaboration ${ }^{\mathrm{b}}$ \\ ${ }^{\text {a } C F N U L, ~ A v . ~ P r o f . ~ G a m a ~ P i n t o ~ 2, ~ P-1699 ~ L i s b o a ~ C o d e x, ~ P o r t u g a l ~}$ \\ b CERN, CH-1211 Geneva 23, Switzerland \\ c Instituto Tecnológico e Nuclear, Estrada Nacional 10, P-2685 Sacavém, Portugal \\ d Hahn-Meitner-Institut Berlin, Glienicker Straße 100, D-14109 Berlin, Germany \\ Received 8 October 1998
}

\section{Abstract}

On-line Perturbed Angular Correlation (PAC) experiments were performed on the $66.0 \mathrm{keV}$ excited state of ${ }^{127} \mathrm{Cs}$, using the $\gamma(114.7 \mathrm{keV})-\mathrm{e}_{\mathrm{L}}^{-}(66.0 \mathrm{keV})$ cascade from the decay of the short-lived ${ }^{127} \mathrm{Ba}\left(T_{1 / 2}=13 \mathrm{~min}\right)$ isotope produced at the ISOLDE facility at CERN. The PAC experiments were performed with an optimized $\gamma-\mathrm{e}^{-}$spectrometer coupled to the ISOLDE beam line, which allowed simultaneous implantation and measurement. The optimization of the experiment is described and the first results on metallic foils and single crystals of $\mathrm{Al}, \mathrm{Be}, \mathrm{Ga}, \mathrm{Zn}$, and $\mathrm{Ni}$ are presented and discussed. The derived nuclear moments of the $66.0 \mathrm{keV}$ excited state of ${ }^{127} \mathrm{Cs}$ are $|\mu|=2.9(2) \mu_{\mathrm{N}}$ and $|Q|=0.58(12) b$. Applications of this new PAC isotope are outlined. (c) 1999 Elsevier Science B.V. All rights reserved.

PACS: 31.30.G; 29.25.R; 29.40.M; 21.10.K

Keywords: On-line production of radioactive isotopes; Ion implantation; Perturbed angular correlations; Hyperfine interactions; Nuclear moments

\section{Introduction}

The application of hyperfine interaction techniques to problems in solid state physics has been steadily growing over the last decade with the increased use of radioactive ion beam facilities, such

\footnotetext{
${ }^{*}$ Corresponding author. Tel.: +351-1790-4987; fax: +3511795-4288; e-mail: soaresjc@alf1.cii.fc.ul.pt
}

as ISOLDE at CERN [1,2]. New applications of the $\mathrm{e}^{-}-\gamma$ Perturbed Angular Correlation (PAC) technique have recently extended the sensibility limits of the $\gamma-\gamma$ PAC method [3] using probe nuclei with highly converted cascades such as ${ }^{73} \mathrm{Ge}$ and ${ }^{197} \mathrm{Hg}$ [4-6]. In order to efficiently use shortlived isotopes (i.e., with $T_{1 / 2}$ smaller than about 30 min) on-line implantation and measurement are essential. Such is the case of the ${ }^{127} \mathrm{Ba} /{ }^{127} \mathrm{Cs}$ probe used in the present work. 
An alkali probe element is extremely interesting to study several problems in solid state research, like diffusion mechanisms looking at the interaction of alkali impurities with implantation defects in ferromagnetic metals [7], or to probe charge density shifts on the $\mathrm{Ba}$ site of high- $T_{\mathrm{C}}$ superconductors [8].

The main goal of the present work is the introduction of ${ }^{127} \mathrm{Cs}$ as a new PAC probe for hyperfine interaction studies. The optimization of the experiment is described, and first results are presented and discussed.

\section{Experimental details}

A partial decay scheme of the short-lived ${ }^{127} \mathrm{Ba}$ isotope is shown in Fig. 1. The aim of the present work is to perform PAC measurements on the 66.0 $\mathrm{keV}$ excited state of ${ }^{127} \mathrm{Cs}$. For this purpose, the $114.7 \mathrm{keV} \gamma$-ray and the L-conversion electrons of the $66.0 \mathrm{keV}$ transition in ${ }^{127} \mathrm{Cs}$ have to be de-

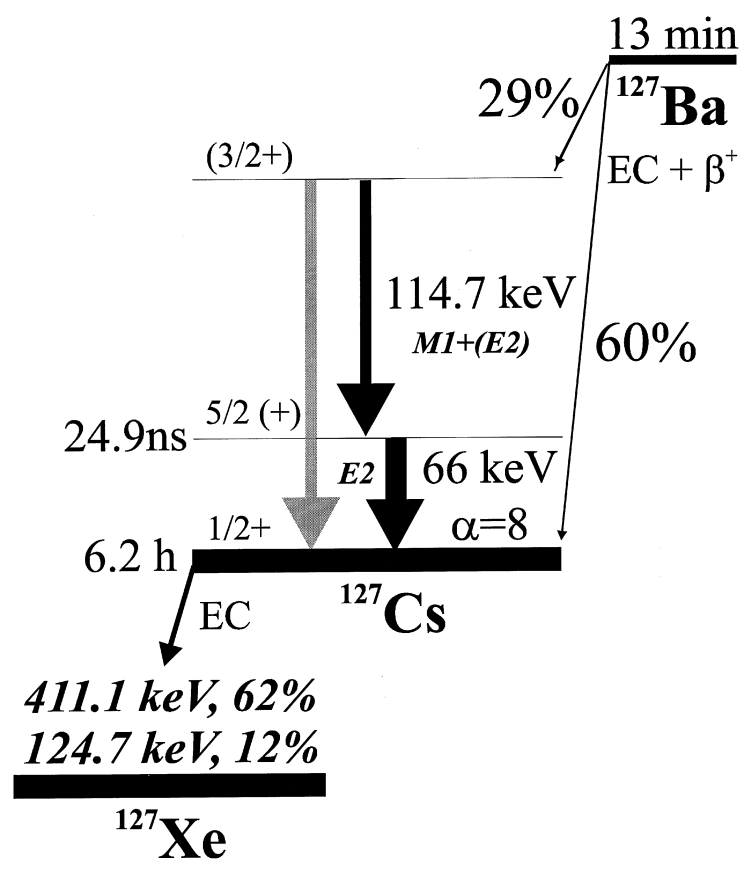

Fig. 1. Simplified decay scheme of the ${ }^{127} \mathrm{Ba} /{ }^{127} \mathrm{Cs}$ decay. The two most intense gamma lines obtained from the ${ }^{127} \mathrm{Cs} /{ }^{127} \mathrm{Xe}$ decay are also indicated. tected, and the time between their detection has to be accurately measured. The conversion electrons are energy discriminated by magnetic lenses prior to the detection on plastic scintillators. This method provides an easy and efficient way to select the electrons of interest from complex decay cascades or from isobaric contamination [9]. Since good time resolution is essential in PAC experiments, cylindrical 1.75 in. diameter and 0.3 in. thick $\mathrm{BaF}_{2}$ scintillators were used for $\gamma$ detection. The small thickness of the $\mathrm{BaF}_{2}$ crystals allows maximum detection efficiency for $\gamma$-rays with energies below $150 \mathrm{keV}$. Once high-count rates can be expected due to the short ${ }^{127} \mathrm{Ba}$ half-life, as well as due to the accumulation of the decay products into the sample, the scintillators were coupled to XP2020Q fast photomultipliers with transistorized bases. During the experiments, the gamma detectors have been often running at average count rates as high as $2 \times 10^{5} \mathrm{~Hz}$.

At ISOLDE, the ${ }^{127} \mathrm{Ba}$ isotope is produced during spallation of a Ta-foil target by the $1 \mathrm{GeV}$ proton beam from the PS-Booster accelerator at

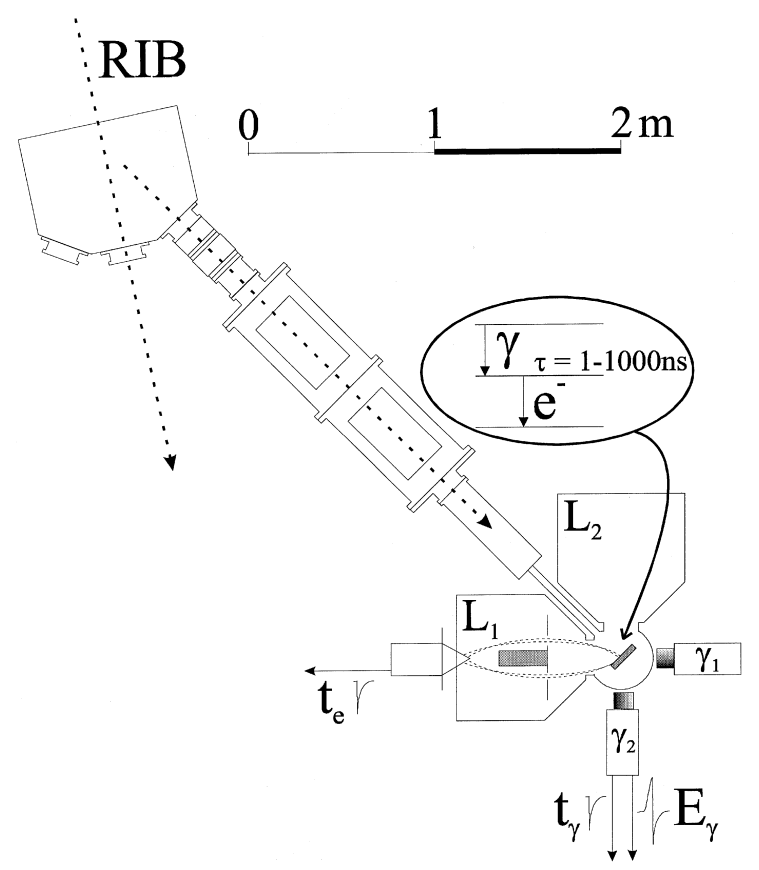

Fig. 2. General view of the $\gamma-\mathrm{e}^{-}$PAC spectrometer mounted on-line with the ISOLDE beam line. 
CERN. A W-surface ion source is coupled to the target and ionizes the alkali, alkali-earth and lanthanide elements. Then a $60 \mathrm{kV}$ extraction electrode produces a beam, which is mass separated by a high resolving power magnet [2].

Due to the ${ }^{127} \mathrm{Ba}$ short half-life, the $\gamma-\mathrm{e}^{-}$PAC spectrometer was coupled to the ISOLDE beam line, as shown in Fig. 2. Conversion electron spectra, such as shown in Fig. 3, were used to check the yields and isobaric contamination of mass 127. It was found that the beam consisted mainly of the ${ }^{127} \mathrm{Ba}^{+}$and ${ }^{127} \mathrm{Cs}^{+}$ions, with yields of $1.0(3) \times 10^{8} \mathrm{p} / \mu \mathrm{C}[10]$ and $5(4) \times 10^{7} \mathrm{p} / \mu \mathrm{C}$, respectively.

Under the above described experimental conditions, 2-4 h of beam time per each PAC spectrum was enough to acquire significant statistics.

\section{Results and discussion}

\subsection{Electron, gamma and half-life spectra}

Fig. 3 shows an electron spectrum taken with the magnetic lens set at its minimum aperture, where all the electron-lines are clearly separated. One notes, in particular, that the $124.7 \mathrm{keV} \mathrm{K}$-line (from the ${ }^{127} \mathrm{Cs} /{ }^{127} \mathrm{Xe}$ decay) appears rather small. The continuously increasing background is due to $\beta^{+}$particles which are emitted during the ${ }^{127} \mathrm{Ba} /$ ${ }^{127} \mathrm{Cs}$ decay. Since to each channel corresponds a

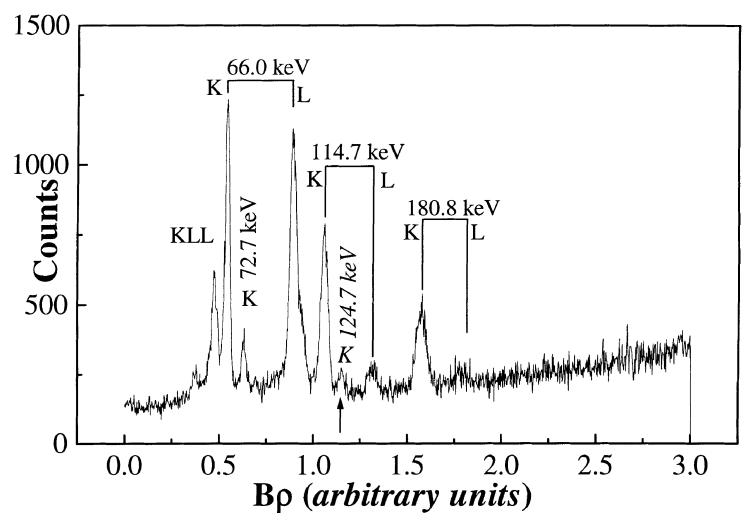

Fig. 3. Electron spectrum made during implantation of the ${ }^{127} \mathrm{Ba} /{ }^{127} \mathrm{Cs}$ beam produced by the $\mathrm{Ta} / \mathrm{W}$ target/ion source (in secular equilibrium). different setting of the magnetic field, an acquisition time of 16 min was necessary to scan all over the conversion electrons energy range with enough statistics.

The electron spectrum as well as the PAC measurements were taken with a constant decay rate of the parent ${ }^{127} \mathrm{Ba}$. This was achieved by performing periodic implantations with an appropriate beam gate duty cycle, in order to reach a convenient secular equilibrium during the measurements. The use of magnetic spectrometers mounted on-line with the ISOLDE beam line allowed to detect the Auger KLL and the $66.3 \mathrm{keV}$ $\mathrm{K}$-conversion electrons lines not previously reported [11-13]. However, a large fraction of such low energy electrons generate pulse signals of small amplitude on the plastic scintillators, of the order of magnitude of the photomultiplier's noise. Since all events are discriminated by a single channel analyzer and this affects in different ways electrons of different energies, the conversion coefficients and thus the multipolarities of the transitions could not be accurately determined.

Fig. 4(a) shows gamma spectra measured with a 2 in. thick $\mathrm{BaF}_{2}$ conical detector commonly used in PAC experiments [9]. The top spectrum (thick line) was measured with the ${ }^{127} \mathrm{Ba}$ activity in secular equilibrium, after $2 \mathrm{~h}$ of continuous implantation. It is clearly seen that the $124.7 \mathrm{keV} \gamma$-lines of the ${ }^{127} \mathrm{Cs} /{ }^{127} \mathrm{Xe}$ decay start mixing up with the $114.7 \mathrm{keV} \gamma$-line of interest from the ${ }^{127} \mathrm{Ba} /{ }^{127} \mathrm{Cs}$ decay. Furthermore, the $411.1 \mathrm{keV}$ and the 511 $\mathrm{keV}$ positron annihilation $\gamma$-lines appear rather intense. The bottom spectrum (thin line) was obtained with beam off after decay of all ${ }^{127} \mathrm{Ba}$. Only the gamma lines that come from the ${ }^{127} \mathrm{Cs} /{ }^{127} \mathrm{Xe}$ decay are seen.

Fig. 4(b) shows two $\gamma$-spectra made after the first implantation shot on a freshly mounted aluminum foil. No $\gamma$-lines from the ${ }^{127} \mathrm{Cs} /{ }^{127} \mathrm{Xe}$ decay was observed at this time. The thin line represents a $\gamma$-spectrum made with the 2 in. thick detector, while the thick line represents a spectrum made with the $0.3 \mathrm{in}$. thick detector. It is clearly seen that, by using the thinner $\mathrm{BaF}_{2}$ detector, only the intensities of the 180.8 and $511 \mathrm{keV}$ gamma lines are strongly attenuated. The use of such thin crystals has as an obvious disadvantage a worst 


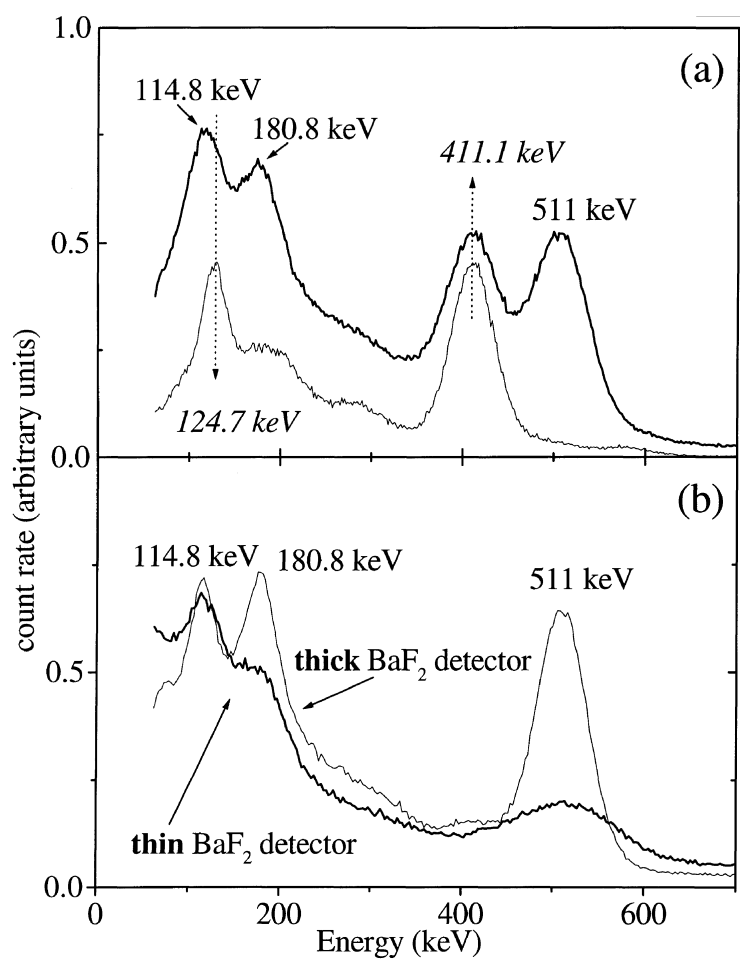

Fig. 4. (a) Gamma spectra measured with a 2 in. thick $\mathrm{BaF}_{2}$ conical detector. The top spectrum (thick line) was measured with the ${ }^{127} \mathrm{Ba}$ activity in secular equilibrium, after $2 \mathrm{~h}$ continuous implantation. The bottom spectrum (thin line) was obtained with beam off after decay of all ${ }^{127} \mathrm{Ba}$. (b) The thin line represents a gamma spectrum made with the 2 in. thick detector after the first implantation shot, on a freshly mounted aluminum foil. The thick line represents a spectrum made with the 0.3 in. thick detector.

energy resolution, of the order of $20 \%$ for the 662 $\mathrm{keV}{ }^{137} \mathrm{Cs}$ line, while $11 \%$ is achieved with the thicker crystal.

Fig. 5 shows the half-life histogram of the 66.0 $\mathrm{keV}$ state, which was obtained by performing time coincidences between the $114.7 \mathrm{keV}$ gamma-ray and the $\mathrm{L}(66 \mathrm{keV})$ conversion electrons. From this histogram we obtained a precise value of the halflife of the $66.0 \mathrm{keV}$ state, $T_{1 / 2}=24.88(30)$. The quoted error includes the error due to the time calibration uncertainty (about 1\%). The resolving time of the experimental setup was measured during the decay of ${ }^{127} \mathrm{Cs}$ to ${ }^{127} \mathrm{Xe}$. By letting the $\gamma$ and electron energy windows set at the same values as during the PAC experiments, a prompt spec-

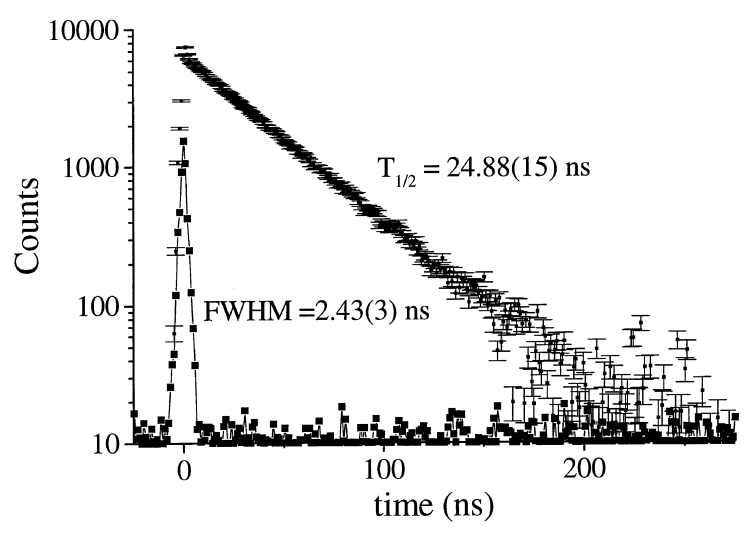

Fig. 5. Measurements of the half-life of the $66.0 \mathrm{keV}{ }^{127} \mathrm{Cs}$ state, and of the time resolution of the experimental setup.

trum with $\mathrm{FWHM}=2.43(3)$ ns was obtained, detecting $\beta^{+}$and inelastically scattered conversion electrons in prompt coincidence with the $124.7 \mathrm{keV}$ $\left({ }^{127} \mathrm{Xe}\right)$ gamma line.

\subsection{The quadrupole interaction of ${ }^{127} \mathrm{Cs}$ in $\mathrm{Al}, \mathrm{Be}$, $\mathrm{Zn}$ and $\mathrm{Ga}$}

Figs. 6-9 show the experimental PAC functions, $R(t)$, measured after implantation of ${ }^{127} \mathrm{Ba}$ in $\mathrm{Al}, \mathrm{Zn}, \mathrm{Be}$, and $\mathrm{Ga}$ hosts, respectively. The theoretical fits to the $R(t)$ functions (continuous line in the figures) were calculated numerically taking into account the full Hamiltonian for the quadrupole hyperfine interaction [14].

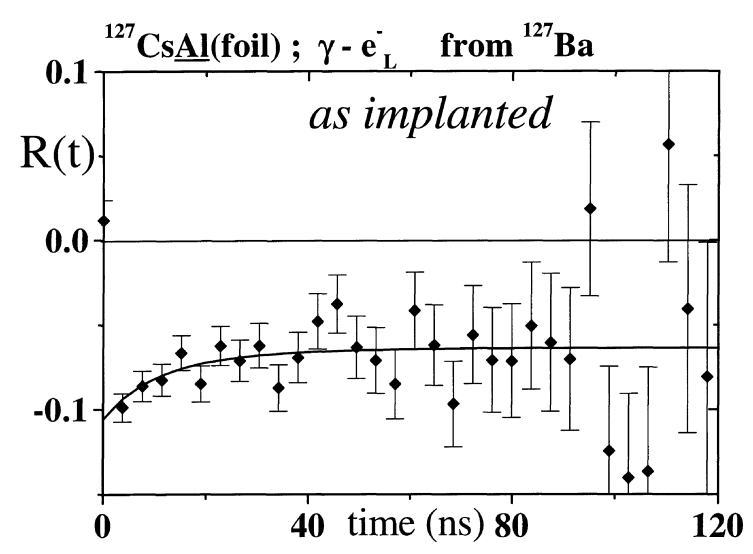

Fig. 6. Experimental $R(t)$ function measured after implantation of ${ }^{127} \mathrm{Ba}$ in an $\mathrm{Al}$ foil at $\mathrm{RT}$. 


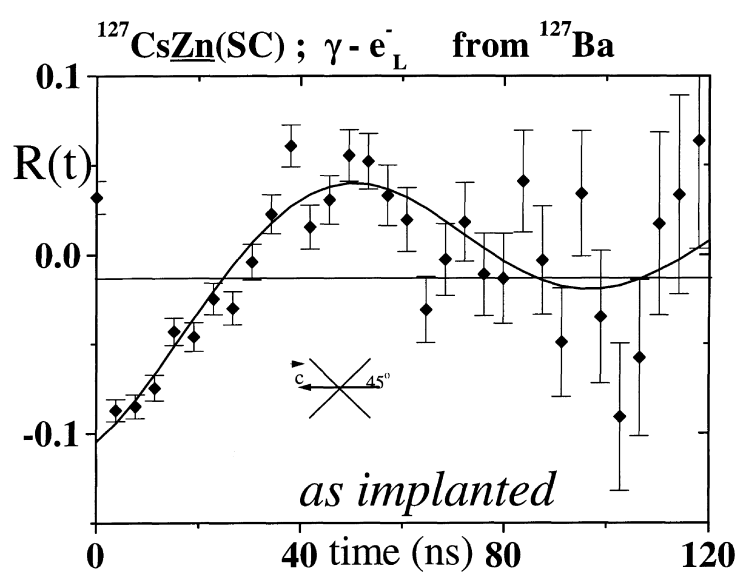

Fig. 7. Experimental $R(t)$ function measured after implantation of ${ }^{127} \mathrm{Ba}$ in a $\mathrm{Zn}$ single crystal at RT. The inset shows the orientation of the $c$-axis in the detector's plane, at $45^{\circ}$ with each detector.

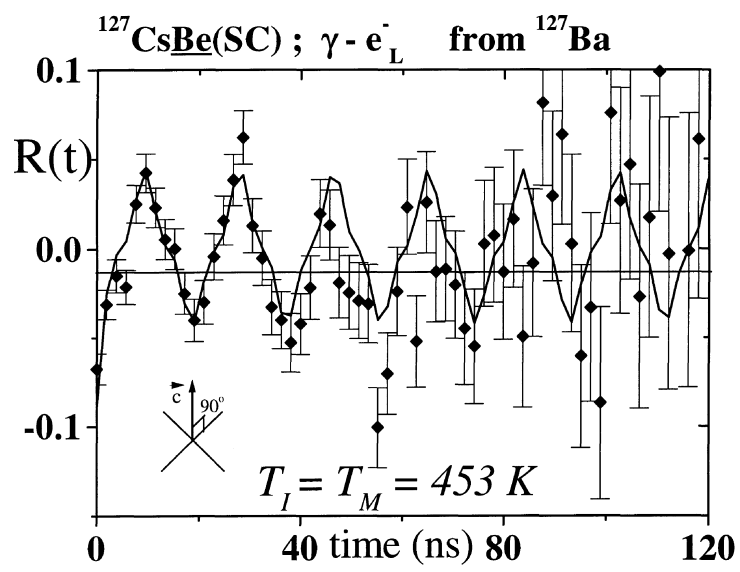

Fig. 8. Experimental $R(t)$ function measured after implantation at $453 \mathrm{~K}$ of ${ }^{127} \mathrm{Ba}$ in a Be single crystal. The insert shows the orientation of the $c$-axis perpendicular to the detector's plane.

Table 1 contains the relevant fit parameters for each spectrum. In the calculation, two independent fractions of probe nuclei, $\left(f_{i}, i=1,2\right)$, each experiencing a quadrupole frequency $v_{Q i}=e Q V_{Z Z}^{i} / \mathrm{h}$ were considered. $V_{Z Z}$ is the principal component of the EFG tensor, which is produced from the extra nuclear charge distribution, and $Q$ is the quadrupole moment of the $66 \mathrm{keV}$ excited state. $f_{1}$ represents the fraction of nuclei that are located in perfect lattice sites, while $f_{2}$ represents the fraction of nuclei that occupy other sites or lattice sites with

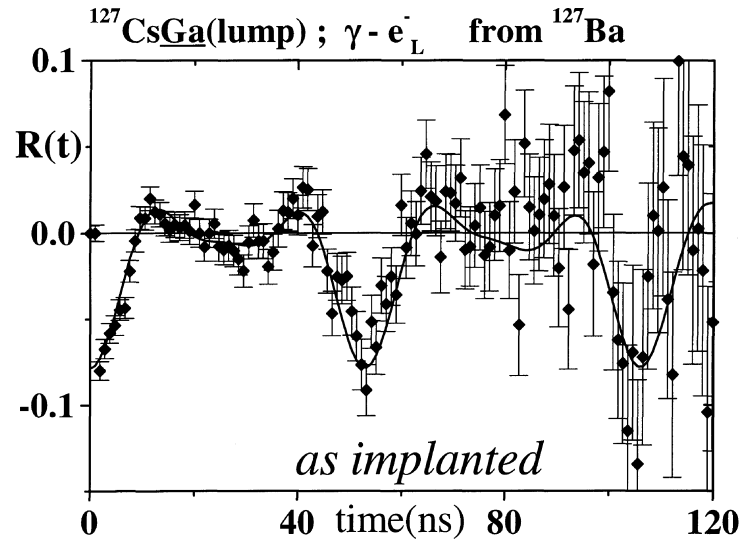

Fig. 9. Experimental $R(t)$ function measured after implantation of ${ }^{127} \mathrm{Ba}$ in Ga lump at RT.

damaged surroundings. For a cascade with an intermediate level with spin $I=5 / 2$, three frequencies are observable per EFG. From these frequencies, the interaction coupling constant, $v_{Q}$, and the asymmetry parameter $\eta=\left(V_{X X}-V_{Y Y}\right) / V_{Z Z}$ are extracted. The interaction with randomly distributed defects does not lead to the measurement of a sharp frequency, but rather to a distribution of frequencies. Here, we assume such a distribution to be of the Lorentzian type, with average value $\left\langle v_{Q}\right\rangle$ and standard deviation $\sigma_{Q}$, depending on the density and variety of the lattice defects.

The amplitude and sign of the $R(t)$ function depend on the anisotropy coefficients of the cascade. For $t=0$, the $R(t)$ function is negative as expected for a $3 / 2 \rightarrow 5 / 2 \rightarrow 1 / 2$ spin sequence. The particle parameter $b_{2}^{\mathrm{L}}(66 \mathrm{keV}, Z=35, \mathrm{E} 2)=1.258$ is positive, and slightly increases the main amplitude coefficient $A_{22}(\gamma-\gamma)=-0.20 \quad[15,16]$. The experimental angular correlation coefficients $A_{k k^{\prime}}^{\exp }\left(\gamma-\mathrm{e}^{-}\right)$are then estimated taking into account the theoretical $A_{k k^{\prime}}^{\mathrm{T}}\left(\gamma-\mathrm{e}^{-}\right)=A_{k k^{\prime}}^{\mathrm{T}}(\gamma-\gamma) \cdot b_{k^{\prime}}$ coefficients, and the $Q(\gamma)_{k} \cdot Q\left(\mathrm{e}^{-}\right)_{k^{\prime}}$ attenuation parameters due to the finite solid angle of the electron and gamma detectors [17]. Another attenuation coefficient $Q^{\text {scatt }}\left(\mathrm{e}^{-}\right)_{k^{\prime}}$ was introduced to account for the elastic electron scattering by the implanted samples [18]. Thus we obtain the experimental coefficients $A_{k k^{\prime}}^{\exp }\left(\gamma-\mathrm{e}^{-}\right)=Q(\gamma)_{k} \cdot Q\left(\mathrm{e}^{-}\right)_{k^{\prime}} \cdot Q^{\text {scatt }}\left(\mathrm{e}^{-}\right)_{k^{\prime}}$. $A_{k k^{\prime}}^{\mathrm{T}} \cdot\left(\gamma-\mathrm{e}^{-}\right)$to be $A_{22}^{\exp }=-0.207, A_{24}^{\text {exp }}=-0.0445$, $A_{42}^{\text {exp }}=0$, and $A_{44}^{\text {exp }}=0$. 
Table 1

Relevant fit parameters obtained for the fits to the experimental $R(t)$ functions as measured in the $\mathrm{Al}, \mathrm{Zn}, \mathrm{Be}$, and $\mathrm{Ga}$ hosts $\left(T_{\mathrm{M}}\right.$ is the measurement temperature)

\begin{tabular}{llcclcrr}
\hline Host & $T_{\mathrm{M}}(\mathrm{K})$ & $f_{1}(\%)$ & $v_{\mathrm{Q} 1}(\mathrm{MHz})$ & $\eta_{1}$ & $f_{2}(\%)$ & $v_{\mathrm{Q} 2}(\mathrm{MHz})$ & $\sigma_{2}(\mathrm{MHz})$ \\
\hline $\mathrm{Al}$ & $\mathrm{RT}$ & $50(9)$ & 0 & 0 & $50(9)$ & 0 & $57(16)$ \\
$\mathrm{Be}$ & 453 & $47(9)$ & $358.8(25)$ & 0 & $53(9)$ & $358.8(25)$ & $1770(142)$ \\
$\mathrm{Zn}$ & $\mathrm{RT}$ & $43(16)$ & $34.5(11)$ & 0 & $57(16)$ & $34.5(11)$ & $21(5)$ \\
$\mathrm{Ga}$ & $\mathrm{RT}$ & 100 & $125.5(7)$ & $0.119(16)$ & - & - & - \\
\hline
\end{tabular}

In the above calculations of the experimental anisotropy coefficients, we assumed that the first transition is pure M1 (no E2 mixing is known) and that the second transition is pure E2. However, the maximum observable amplitude of the $R(t)$ spectra is smaller than the expected by a factor of 2 . Factors such as (a) the contribution of the elastically back-scattered electrons (not easy to calculate), and (b) a 1-2\% E2 mixture in the M1 114.8 $\mathrm{keV}$ transition, are possible sources of such extra reduction factor. On the other hand, the same effect can be explained by the existence of a large fraction of nuclei implanted in highly lattice damaged sites, that generates a large distribution of EFGs which strongly attenuates the $R(t)$ function in the first channels. Since we cannot choose among the different possibilities, we establish for analysis purposes an effective maximum amplitude of the $R(t)$ function by assuming $A_{22}^{\text {eff }}=0.5 \times A_{22}^{\exp }$. Thereafter, the fractions $f_{1}+f_{2}=100 \%$ are normalized to $A_{22}^{\text {eff }}$.

After room temperature (RT) implantation in an Al foil (Fig. 6), about $f_{1}=50(9) \%$ of the ${ }^{127} \mathrm{Ba} /$ ${ }^{127} \mathrm{Cs}$ nuclei are in unperturbed cubic $(\mathrm{EFG}=0)$ lattice sites, which are characterized by $\left\langle v_{Q}\right\rangle=0$ and $\sigma_{Q}=0$. The remainder nuclei are interacting with a wide distribution of defects, characterized by $\left\langle v_{Q}\right\rangle=0$ and $\sigma_{Q}=57(16) \mathrm{MHz}$. Similar results are obtained after implantation at $453 \mathrm{~K}$ in the $\mathrm{Be}$ hcp single crystal (Fig. 7). About $f_{1} \sim 47(9) \%$ ) of the ${ }^{127} \mathrm{Ba} /{ }^{127} \mathrm{Cs}$ nuclei take up the same unique position characterized by $\left|v_{Q 1}\right|=358.8(25) \mathrm{MHz}$ and $\eta_{1}=0$, without defects in their neighborhood $\left(\sigma_{Q 1}=0\right)$. The rest of the Ba nuclei are in a highly damaged environment or in several defect-associated positions of the Be lattice, characterized by $\left\langle v_{Q 2}\right\rangle=v_{Q 1}$, and $\sigma_{Q 2}=1770(142) \mathrm{MHz}$.
A completely different behavior was found after RT implantation into $\mathrm{Zn}$ and Ga. In a $\mathrm{Zn}$ hep single crystal, $100 \%$ of the ${ }^{127} \mathrm{Ba} /{ }^{127} \mathrm{Cs}$ nuclei reside at the same lattice site, which is characterized by $\left|v_{Q 1}\right|=34.5(11) \mathrm{MHz}$ and $\eta_{1}=0$ (Fig. 8). About $f_{2} \sim 57(16) \%$ of these nuclei interact with a distribution of defects characterized by $\left\langle v_{Q 2}\right\rangle=v_{Q 1}$ and $\sigma_{Q 2}=21(5) \mathrm{MHz}$. This fraction is responsible for the slight damping seen in the $R(t)$ function. After RT implantation in a Ga lump (Fig. 9), 100\% of the ${ }^{127} \mathrm{Ba} /{ }^{127} \mathrm{Cs}$ nuclei are found in the same site of the orthorhombic lattice, characterized by $\left|v_{Q 1}\right|=125.5(7) \mathrm{MHz}$ and $\eta_{1}=0.119(16)$.

\subsection{The magnetic interaction of ${ }^{127} \mathrm{Cs}$ in $\mathrm{Ni}$}

In an attempt to measure the magnetic hyperfine interaction, ${ }^{127} \mathrm{Ba}$ was implanted into the ferromagnetic host Ni. From the spectrum measured in

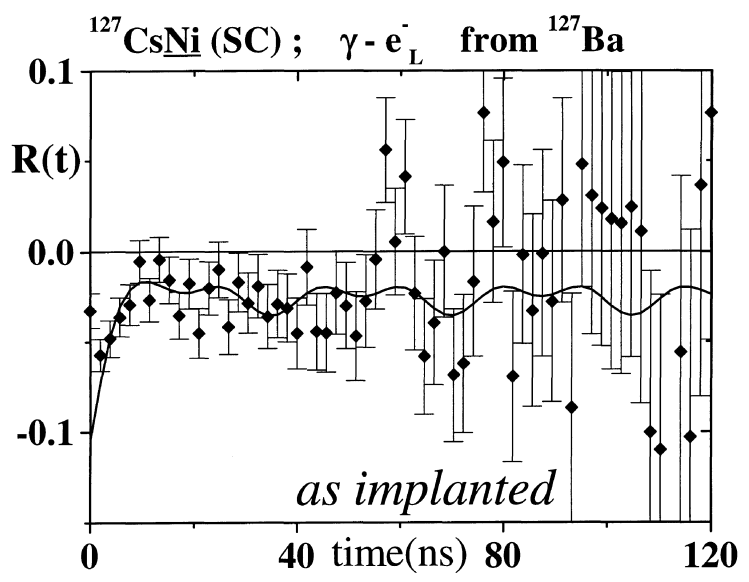

Fig. 10. Experimental $R(t)$ function measured after implantation of ${ }^{127} \mathrm{Ba}$ in a Ni single crystal at RT. 
the as-implanted state (Fig. 10) we could conclude that already $25 \%$ of the implanted nuclei are located in one unique site, with a corresponding Larmor frequency $\left|\omega_{\mathrm{L}}\right|=184(20) \mathrm{Mrad} \mathrm{s}^{-1}$. This preliminary experiment should therefore be continued performing measurements as a function of temperature to recover the damage after implantation.

\section{Discussion}

The $R(t)$ spectra measured in the non-cubic metallic $\mathrm{Be}, \mathrm{Zn}$ and $\mathrm{Ga}$ hosts have clearly shown a characteristic set of three observable frequencies per EFG. This fact proves that the spin of the 66.0 $\mathrm{keV}$ state is $I=5 / 2$ [3].

The nuclear quadrupole moment can be estimated in two different ways. The first one is based on a point charge calculation of the ionic EFG contribution, $V_{Z Z}($ ion $)=-1.35 \times 10^{16} \mathrm{~V} \mathrm{~cm}^{-2}$, produced by the Be lattice at the octahedral interstitial position, where the implanted $\mathrm{Ba}$ is known to be located [19]. Then the electronic contribution to the total EFG can be estimated using the empirical "Universal Correlation" [20] in the form $V_{Z Z}=$ $(1-K)\left(1-\gamma_{\infty}\right) V_{Z Z}$ (ion). In this equation $\gamma_{\infty}$ is the antishielding Sternheimer factor that accounts for the polarization of the electronic core, and $K$ is a factor in the range of 2-3 for most probes [21]. Adopting $K=2.5$ and $\gamma_{\infty}\left(\mathrm{Cs}^{+1}\right)=-121.3$, we obtain $V_{Z Z}=+2.48 \times 10^{18} \mathrm{~V} \mathrm{~cm}^{-2}$. Then the value $|Q|=0.60(1) b$ is obtained from the measured $\left|v_{Q 1}\right|$ coupling constant in Be. The quoted error includes only the experimental uncertainty of $\left|v_{Q 1}\right|$.

When the same type of calculation is performed for $\mathrm{Zn}$, assuming that $\mathrm{Ba}$ goes to a substitutional position in the $\mathrm{Zn}$ lattice, an unrealistic value of the EFG is obtained. However, there is evidence for an anomalously low EFG value for the alkali elements $\mathrm{Rb}$ in $\mathrm{Zn}$, far away from any first order systematics [22].

The second calculation we performed is based on the known value of the quadrupole interaction of an excited state of ${ }^{80} \mathrm{Rb}$ in $\mathrm{Ga}, v_{Q}=50.8(1)$ $\mathrm{MHz}$ with $\eta=0.283(5)$ [23], obtained with the Angular Distribution technique. The quadrupole moment of the $561 \mathrm{keV}$ state where the measurement is performed, has been determined as
$\left|Q\left({ }^{80} \mathrm{Rb}, 561 \mathrm{keV}\right)\right|=0.51(5) b$ [24]. Since $\mathrm{Rb}$ is isovalent to $\mathrm{Cs}$, we can assume that $V_{Z Z}(\mathrm{CsGa}) /$ $V_{Z Z}(\mathrm{RbGa})=\left(1-\gamma_{\infty}(\mathrm{Cs})\right) /\left(1-\gamma_{\infty}(\mathrm{Rb})\right)$. We thus obtain $\left|Q\left({ }^{127} \mathrm{Cs}\right)\right|=0.58(12) b$. The magnitudes of both values found for $Q$, which were obtained in two independent ways, are in excellent agreement. It is interesting to note that ${ }^{127} \mathrm{Cs}$ has a quadrupole moment similar to the ground state of ${ }^{131}$ Cs [25]. This observation may be explained by the fact that both nuclear states should have a similar singleparticle configuration.

The estimation of the nuclear magnetic moment $(\mu)$ of the $66 \mathrm{keV}$ state has been made taking together the known hyperfine field $B_{\mathrm{hf}}=3.5(5) \mathrm{T}$ for $\mathrm{Cs}$ in $\mathrm{Ni}$ [26], and the value now measured for the Larmor frequency $\left|\omega_{\mathrm{L}}\right|=\left|2 \pi g \mathrm{~B}_{\mathrm{hf}} \mu_{\mathrm{N}} / h\right|=184(20)$ $\mathrm{Mrad} \mathrm{s}^{-1}$. Then a magnetic moment of $|\mu|=2.7(5)$ $\mu_{\mathrm{N}}$ is found, also close to the magnetic moment of the ${ }^{131} \mathrm{Cs}$ ground state [25].

\section{Conclusions}

In this work, we have shown that the $66 \mathrm{keV}$ state in ${ }^{127} \mathrm{Cs}$ can be used as a probe state of a new PAC element. For these experiments, the $\gamma-\mathrm{e}^{-}$ PAC spectrometer has been mounted on-line with the ISOLDE beam line. The combination of a Ta target with $\mathrm{W}$ ion-source was found to be the best option to produce cleans ${ }^{127} \mathrm{Ba}$ beams at ISOLDE. Thin $\mathrm{BaF}_{2}$ gamma detectors were installed for optimized efficiency at low energy and high count rate. From the PAC measurements, the half-life, spin, and nuclear quadrupole moment of the 66 $\mathrm{keV}$ excited state ${ }^{127} \mathrm{Cs}$ were derived. A first hint on the magnetic moment of this state is presented.

The applications foreseen for this new PAC probe element involve investigations of Ba-containing high- $T_{\mathrm{c}}$ superconductors, point defects studies in metals with alkali-earth elements, and also the study of amorphous-crystalline phase transitions in barium materials.

\section{Acknowledgements}

This work was partially funded by FCT, Portugal, through projects CERN/1082/96, CERN/ 
1156/97, PBICT/C/CTM/1891/9 and grants under the PRAXIS XXI Programme (J.G.C. and J.G.M.).

\section{References}

[1] J.G. Correia, Nucl. Instr. and Meth. B 136-138 (1998) 736.

[2] E. Kugler, D. Fiander, B. Jonson, H. Haas, A. Przewloka, H.L. Ravn, D.J. Simon, K. Zimmer, Nucl. Instr. and Meth. B 70 (1992) 41.

[3] Th. Wichert, N. Achziger, H. Metzner, R. Sielemann, in: G. Langouche (Ed.), Hyperfine Interaction of Defects in Semiconductors, Elsevier, Amsterdam, 1992.

[4] J.G. Correia, H. Haas, J.G. Marques, A.A. Melo, J.C. Soares, ISOLDE Collaboration, Hyp. Int. 80 (1993) 1321.

[5] J.G. Marques, J.G. Correia, A.A. Melo, J.C. Soares, E. Alves, M.F. da Silva, ISOLDE Collaboration, J. Appl. Phys. 76 (1994) 6906.

[6] J.G. Correia, J.G. Marques, E. Alves, D. Forkel-Wirth, S.G. Jahn, M. Restle, M. Dalmer, H. Hofsäss, K. Bharuth-Ram, ISOLDE Collaboration, Nucl. Instr. and Meth. B 127/128 (1997) 723.

[7] M. Lindroos, H. Haas, J. de Wachter, H. Pattyn, G. Langouche, Nucl. Instr. and Meth. B 64 (1992) 256.

[8] R. Gatt, J.S. Olsen, L. Gerward, I. Bryntse, A. Kareiva, I. Panas, L.G. Johansson, Phys. Rev. B 57 (1998) 13922.

[9] J.G. Marques, J.G. Correia, A.A. Melo, M.F. da Silva, J.C. Soares, Nucl. Instr. and Meth. B 99 (1995) 645.
[10] The notation " $\mathrm{p} / \mu \mathrm{C}$ " means particles (in-beam) produced per $\mu$-Coulomb of the $1 \mathrm{GeV}$ proton beam..

[11] T.W. Conlon, Nucl. Phys. A 161 (1971) 289.

[12] B.P. Pathak, I.L. Preiss, Phys. Rev. C 11 (1975) 1762.

[13] G. Beyer, A. Jasinski, O. Knotek, H.-G. Ortlepp, H.-U. Siebert, R. Arlt, E. Herrmann, G. Musiol, H. Tyrroff, Nucl. Phys. A 260 (1976) 269.

[14] N.P. Barradas, M. Rots, A.A. Melo, J.C. Soares, Phys. Rev. B 47 (1993) 8763.

[15] R.S. Hager, E.C. Seltzer, Nucl. Data A 4 (1968) 1.

[16] R.S. Hager, E.C. Seltzer, Nucl. Data A 4 (1968) 397.

[17] M.J.L. Yates, in: K. Siegbahn (Ed.), Alpha-, Beta- and Gamma-ray Spectroscopy, vol. 2, North-Holland, Amsterdam, 1965, p. 1691.

[18] S. Frankel, Phys. Rev. 83 (1954) 673.

[19] R. Vianden, E.N. Kaufmann, J.W. Rodgers, Phys. Rev. B 22 (1980) 63.

[20] R.S. Raghavan, P. Raghavan, E.N. Kaufman, Phys. Rev. Lett. 34 (1975) 1280.

[21] F.D. Feiock, W.R. Johnson, Phys. Rev. 187 (1969) 39.

[22] P. Raghavan, private communication.

[23] M. Menningen, H. Grawe, H. Haas, W.-D. Zeitz, R. Keitel, in: Ann. Rep. Hahn-Meitner Institut, Berlin, 1979, p. 80.

[24] Ch. Stenzel, Ph.D. Thesis, Freie Universitaet Berlin, 1986.

[25] P. Raghavan, Atomic Data and Nucl. Data Tables 42 (1989) 189.

[26] F. Namavar, M. Rots, R. Coussement, H. Ooms, J. Claes, Hyp. Int. 4 (1978) 716. 